\title{
Profile of Organic Acid Concentrations in the Digestive Gland and Hemolymph of Biomphalaria glabrata under Estivation
}

\author{
José Clecildo Barreto Bezerra/ ${ }^{+}$, Andreas Kemper*, \\ Wilhelm Becker*
}

\begin{abstract}
Setor de Parasitologia, Instituto de Patologia Tropical e Saúde Pública, Universidade Federal de Goiás, Rua Delenda Resende de Melo, s/nº, Setor Universitário, 74605-050 Goiânia, GO, Brasil *Zoologisches Institut und Museum, Universität Hamburg, Martin-Luther-King-Platz, 20146 Hamburg, Germany
\end{abstract}

Using high performance liquid chromatography (HPLC) analysis it was possible to determine simultaneously the concentration of organic acids (pyruvate, lactate, succinate, fumarate, malate, acetate, propionate, acetoacetate, and $\beta$-hydroxybutyrate) in the digestive gland and the extracellular concentration of these same acids in the hemolymph of estivating Biomphalaria glabrata, the intermediate host of Schistosoma mansoni. After a 7 day period of estivation, there was a significant increase in the tissue levels of lactate, succinate, malate and acetate compared to non-estivating snails. After 14 days of estivation, the levels of lactate and acetate were also significantly elevated. The hemolymph concentrations of pyruvate and acetate increased significantly after 7 days and acetate concentrations continued to be significantly increased up to 14 days of estivation. The other organic acids studied, such as ketone body acetoacetate and $\beta$-hydroxybutyrate or the volatile acid propionate, did not accumulate. Their tissue concentrations, however, increased on the 7th day of estivation and reached normal levels within two weeks of estivation for some of them. One should take into consideration how the reduction in metabolism can be handled under aerobic conditions, and what role anaerobic pathways may play in both energy formation and redox balance processes.

Key words: Biomphalaria glabrata - estivation - high performance liquid chromatography - organic acid Schistosoma mansoni

Estivation is a natural phenomenon of ecological importance which allows snails and other animal groups to become inactive and dormant in response to drought (Schmidt-Nielsen 1990). Such behavior allows these animals to survive long periods of unfavorable conditions, as is the case for the freshwater snail Biomphalaria glabrata, the intermediate host of Schistosoma mansoni. In the estivating state, $B$. glabrata can survive for up to seven months without water (Barbosa \& Barbosa 1958).

Estivation is of epidemiologic importance since the parasite is able to survive for long periods in its larval stage in estivating snails, thereby maintaining its chances of returning to an aquatic enviroment to resume its normal life cycle once favorable conditions reappear (Richards 1967).

This study was supported in part by $\mathrm{CNPq}$, Brazil and DAAD, Germany.

${ }^{+}$Corresponding author. Fax +55-62-202.3066. E-mail: clecildo@ufg.br

Recevied 28 May 1999

Accepted 28 July 1999
During estivation, food uptake ceases, water loss occurs and the snails are not able to rid themselves of their excretion products in a usual way. Gas exchange may also be affected. All of these alterations exert an influence on the snail's metabolism which may be reflected in the concentration of metabolites.

Under conditions of drought, freshwater snails require a metabolic strategy to cover their energy requirements which are generally decreased. This may be interpreted as a homeostatic stabilization. In freshwater and land snails, adaptive changes in metabolism occur, such as phosphorylation and changes in the kinetic parameters and activity of glycolytic enzymes (Ellersiek 1976, Brooks \& Storey 1996). Changes in protein phosphorylation can be observed in the land snails Otala lactea on the basis of changes in the activities of protein kinases and protein phosphatases, regulated by changes in second messenger levels (Brooks \& Storey 1996). As reported by Storey (1996), reactivation of land snails after 30 days of estivation causes $\mathrm{O}_{2}$ consumption to be twice higher than during the steady state of the snail. Pyruvate kinase activity is reduced while pyruvate dehydrogenase activity is 
fully reactivated within 60 min (Herreid 1977, Whitwam \& Storey 1990, Brooks \& Storey 1992), with the glycolytic carbon flow being again carried to the mitochondria through this pathway. Since organic acids play a central role in intermediate metabolism, their tissue and hemolymph concentrations were studied in parallel in estivating snails and compared with those of non-estivating animals. In this context, organic acids may serve as indicators of various metabolic reactions since they represent important components of energy and intermediate metabolism. Thus, they may indicate the use of carbohydrates as an energy source in the flow of aerobic and, in the case of estivating molluscs, anaerobic carbon, the replacement of glucose through gluconeogenesis, and of protein via glucogenic amino acids or metabolism of lipids on a smaller scale via fatty acids and ketone bodies.

Using high performance liquid chromatography (HPLC) analysis of one sample, it was possible to determine simultaneously the concentration of nine organic acids in the digestive gland and hemolymph of the snail. Using such data, one can then evaluate in a broader manner the physiological processes that permit the snail to survive under adverse environmental conditions.

\section{MATERIALS AND METHODS}

Standard conditions - Adult B. glabrata (albino strain, Puerto Rico, 30 per aquarium) were kept in 301 polyethylene aquaria at a water temperature of $26 \pm 2^{\circ} \mathrm{C}$ under constant aeration and on an artificial $12 \mathrm{hr}$ photoperiod. The water was replaced continuously with fresh tap water. The snails were fed ad libitum with standard snail food according to Standen (1951) as modified by Becker and Lamprecht (1977). The aquaria were cleaned weekly for the removal of feces and mucus. The shell diameter of the snails used in these experiments was $18 \pm 2 \mathrm{~mm}$.

Estivation - The snails were kept in an acrylic frame hung above a water surface. The air relative humidity was $85 \%$.

Sample preparation - The digestive glands and hemolymph were obtained from the snails after 7 and 14 days of estivation and prepared and assessed according to standard conditions.

Digestive gland - The snails were placed on a petri dish containing isotonic buffer. The shell was carefully broken and the soft tissues dissected out. The digestive gland was assessed and the intestine removed. The tissues were frozen in liquid nitrogen and then transferred to a glass vessel containing $12 \%$ perchloric acid (PCA) at $5^{\circ} \mathrm{C}$. Subsequently, the organs were homogenized, sonicated and centrifuged at $12,000 \mathrm{~g}$ for $10 \mathrm{~min}$ at $2^{\circ} \mathrm{C}$. The supernatant was decanted and neutralized with
$\mathrm{KOH}$ to $\mathrm{pH}$ 7.0, after which a second centrifugation identical to the previous one was performed. The organic acids were finally extracted from the supernatant using Bond-Elut columns (Rumsby et al. 1987, Bezerra et al. 1997). Each sample assayed represented the supernatant of a single digestive gland and was applied to the extraction column in a final volume of $5 \pm 0.5 \mathrm{ml}$.

Hemolymph sampling - The snail's shell was first cleaned with a paper towel and the hemolymph was collected with a Pasteur pipette inserted through a tiny hole made in the shell above the pericardial region. For each group or treatment the hemolymph from three snails was pooled and then centrifuged at $120 \mathrm{~g}$ for $5 \mathrm{~min}$ at $2^{\circ} \mathrm{C}$ in order to remove the hemocytes and cell debris. The resulting supernatant was then used in the analyses described below. A total of ten samples from each group were examined.

Organic acid extraction, identification and quantification by HPLC - The organic acids were extracted from the previously prepared digestive gland and from the centrifuged hemolymph using Bond-Elut ${ }^{\circledR}$ columns (SAX anion exchangequartenary amine, Analytichem International, Habor City, USA) as described by Bezerra et al. (1997). Under vacuum, the columns were consecutively activated with $1 \mathrm{ml}$ of $0.5 \mathrm{M} \mathrm{HCl}, 1 \mathrm{ml}$ of methanol and $2 \mathrm{ml}$ of HPLC-grade water. They were then loaded with $200 \mu \mathrm{l}$ of hemolymph or with the supernatant of a single digestive gland extract and then with $2 \mathrm{ml}$ of water. For elution, the columns were removed from the vacuum pump and $250 \mu \mathrm{l}$ of 0.5 $M$ sulfuric acid was applied in order to elute the organic acids retained in the column matrix. The eluate was then centrifuged at $1,200 \mathrm{~g}$ for $5 \mathrm{~min}$ and stored at $-70^{\circ} \mathrm{C}$ until analyzed by HPLC. Chromatography was performed at room temperature using a BIORAD-Aminex Ion Exclusion HPX-87H column $(300 \times 7.8 \mathrm{~mm})$ specific for the separation of organic acids. The separation column was protected by a BIORAD-Aminex HPX-85 guard column. The mobile phase was sulfuric acid $(0.5 \mathrm{mM})$ delivered at a flow rate of $0.8 \mathrm{ml} / \mathrm{min}$. The elution profile was determined at $210 \mathrm{~nm}$. The injected volume was $100 \mu$.

The HPLC system was separately calibrated with the acids to be studied and a standard curve was constructed for the acids pooled in a standard solution. The recovery of the acids from the BondElut ${ }^{\circledR}$ extraction column and from the chromatographic separation column was calculated to permit the calculation of their final concentration in the samples analyzed. The calibration of the equipment was assessed daily using the standard solution in order to determine the retention time and concentration of the acids under study. 
The statistical calculations were based on the non-parametric Wilcoxon and Mann-Whitney tests (U-test) (Sachs 1990). The differences were considered to be significant when $\mathrm{P} \leq 0.05$.

\section{RESULTS}

A total of 60 samples obtained under estivating and non-estivating conditions were examined.

During the first days of estivation, feces were produced normally. As the experiment progressed, feces production diminished. No ovipositing occurred during the observation period and after the fourth day, the snails remained retracted inside their shells. The concentration of nine organic acids in the digestive gland and hemolymph of $B$. glabrata was determined simultaneously by HPLC after 7 and 14 days of estivation.

Fig. 1 shows that pyruvate was the end product of glycolysis and of lactate, an indicator of anaerobic metabolism. The pyruvate concentration in hemolymph was increased after 7 days. The lactate level increased in the homogenates of the digestive gland from estivating animals.

Other end products of a possibly anaerobic metabolism, such as succinate, and other elements of the Krebs cycle, such as fumarate and malate, were decreased in the hemolymph until the end of the experiment. The levels of malate remained unchanged in the end of study period in tissue (Fig. 2).
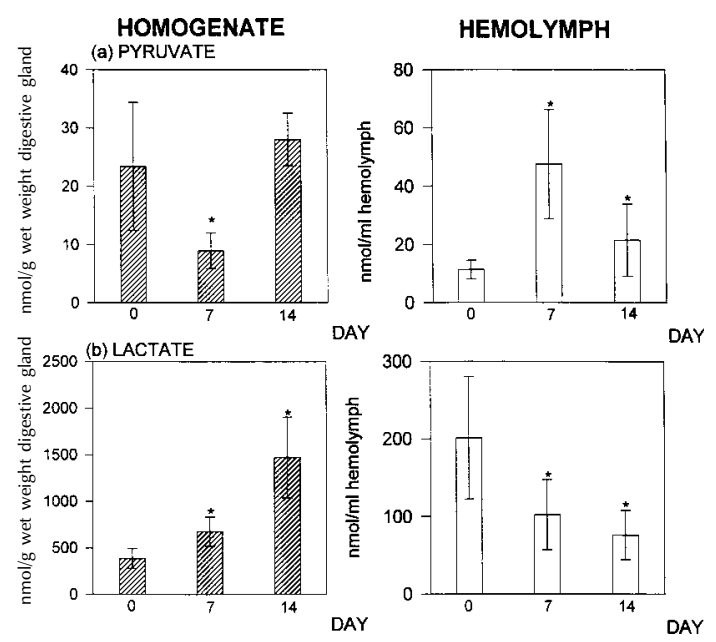

Fig. 1: organic acid concentration of pyruvate (a) and lactate (b) in the digestive gland and in the hemolymph of Biomphalaria glabrata under estivation. The numbers of experiment per group were $n=10$. The values shown are the mean + S.D. $* \mathrm{P} \leq 0.05$ compared to non-estivating snails (U-test).
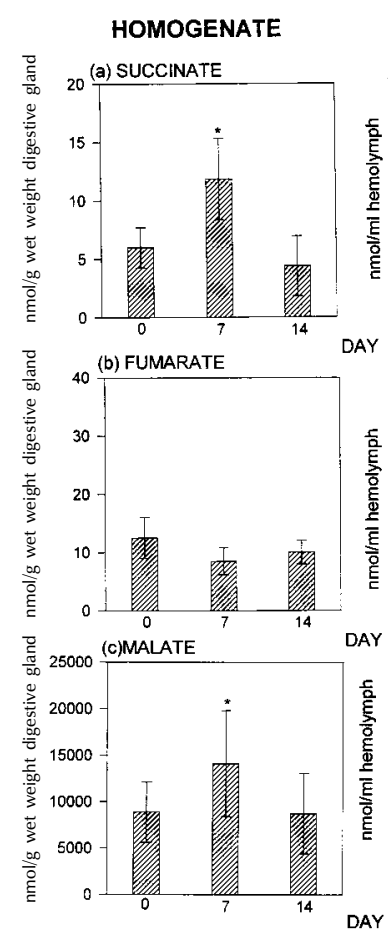

HEMOLYMPH
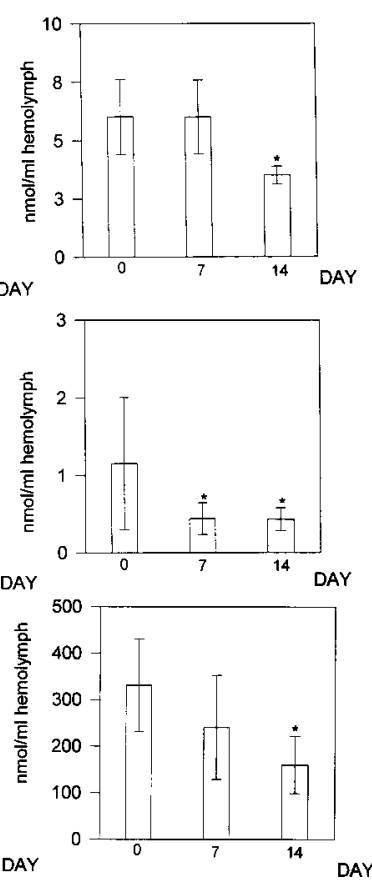

Fig. 2: succinate (a), fumarate (b) and malate (c) in the digestive gland and in the hemolymph of Biomphalaria glabrata under estivation. The numbers of experiment per group were $n=10$. The values shown are the mean + S.D. $* \mathrm{P} \leq 0.05$ compared to non-estivating snails (U-test).

The concentrations of volatile acids (Fig. 3) such as propionate was reduced in the hemolymph, but was normal in the digestive gland. A marked increase was noted in intra- and extracellular acetate concentrations.

The levels of other indicators of lipid metabolism, such as the ketone bodies acetoacetate and ß-hydroxybutyrate, were decreased (Fig. 4).

\section{DISCUSSION}

Under estivation, there is a marked reduction in waste excretion, followed by water loss from the body surface. Epiphragm formation by the snails in order to ameliorate the drying effect of estivation was not observed in the present study or in others (von Brand \& Mehlman 1953), in contrast to what occurs in land molluscs.

Estivation reduces the snails' movements with a consequent decrease in their metabolic activity. In the case of B. glabrata, the latter may be reduced by at least $80 \%$ (Becker 1980). Estivation implies that complex physiological changes need to be studied since this behavior induces a fasting state as a result of the progressive decrease of feed- 

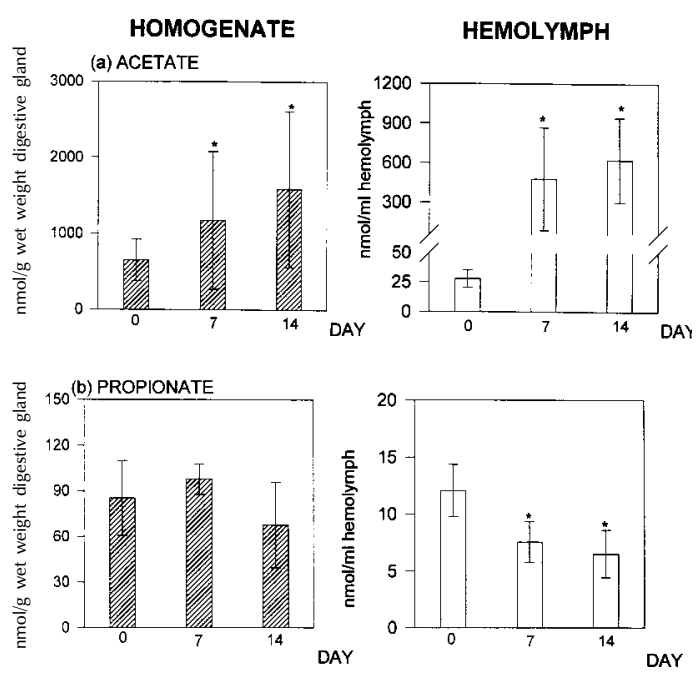

Fig. 3: volatile acid concentration, i.e., acetate (a) and propionate (b), in the digestive gland and in the hemolymph of Biomphalaria glabrata under estivation. The numbers of experiment per group were $\mathrm{n}=10$. The values shown are the mean + S.D. $* \mathrm{P} \leq 0.05$ compared to non-estivating snails (U-test).
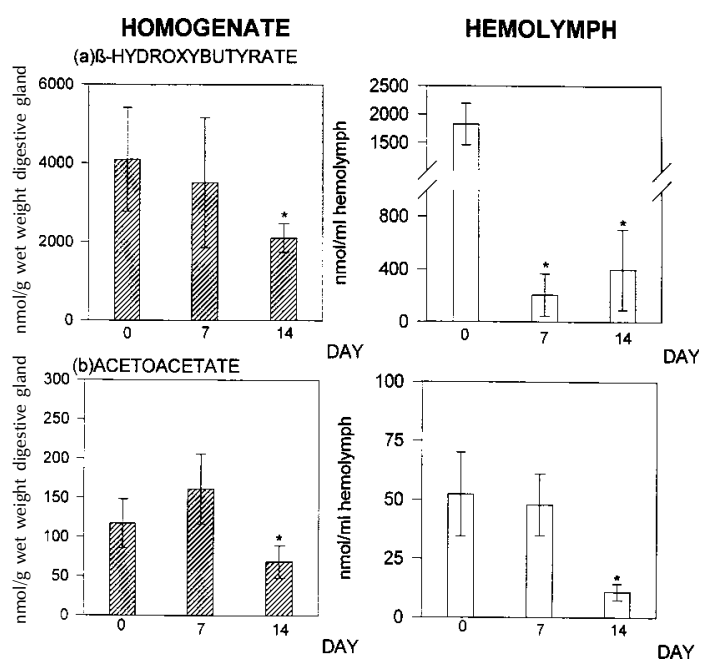

Fig. 4: concentration of the ketone bodies $\beta$ hydroxybutyrate (a) and acetoacetate (b) in the digestive gland and in the hemolymph of Biomphalaria glabrata under estivation. The numbers of experiments per group were $\mathrm{n}=10$. The values shown are the mean + S.D. $* \mathrm{P} \leq 0.05$ compared to non-estivating snails (U-test).

ing and reduced oxygen consumption. Due to the retraction of the snail inside its shell, only a small surface area is available for gas exchange.

The maintenance of pyruvate concentration at the levels observed in non-estivating snails and the significant increase of lactate levels in tissue may reflect an increase in anaerobic metabolism via intensified glycolytic processes. However, an increase in pyruvate and lactate may also result from an enhanced protein catabolism, leading to an increase in glucogenic amino acids (Hochachka 1983) and finally to an increase of these two organic acids. Amino acid metabolism can increase the concentration of urea, as reported for estivating $B$. glabrata (Ellersiek 1976). Similar results obtained with other estivating molluscs were published by De Jorge and Petersen (1970) and Horne (1979).

Athough the possibilities of excretion are greatly reduced under estivation, the concentrations of the components of the tricarboxylic acid cycle analyzed here (succinate, fumarate, and malate) did not increase. Thus, the increase in tissue succinate concentration may indicate a gain in energy via anaerobic pathways. On the 14th day there was a decrease in energy needs as a result of a reduction in metabolic activities. This agrees with the low oxygen consumption reported by von Brand and Mehlman (1953) for estivating $B$. glabrata. Thus, with the reduction in basal metabolism on the 14th day of incubation, the aerobic pathways were still able to maintain an appropriate level of metabolism. This is confirmed by the fact that the intracellular concentrations of malate, succinate and fumarate were within the normal range. This situation was accompanied by a reduction in the hemolymph concentration of these three acids, and reflects their uptake to maintain intracellular.

One of the most interesting results was the significant increase in acetate in the digestive gland as well as in the hemolymph compared with nonestivating snails. This may reflect the oxidative decarboxylation of pyruvate to acetyl-CoA which can lead to the generation of free acetate and ATP via acetylphosphate, thereby inducing partial anaerobic metabolism in estivating $B$. glabrata. The formation of acetate probably resulted not only from a decrease in oxygen consumption, but also from an imbalance in the redox system. However, if this were the case, we would expect an accumulation of other final products of such "anaerobic metabolism", which in fact did not occur until the 14 th day of incubation. The end products of anaerobic pathways, e.g. succinate, lactate and propionate, are well known in molluscs (Wieser 1980, Wolmarans 1987), and we would therefore expect an increase in organic acid levels. However, this was not the case. Thus, it is still unclear which metabolic pathways account for the accumulation of acetate. An increase in the level of acetate (a volatile acid) is not believed to be very toxic for cells (Mehlman \& von Brand 1951) and may therefore represent an advantage for estivating snails since this compound may easily be excreted across the body surface by invertebrates (Bryant 1993). 
The rise in acetate concentration may also indicate a corresponding rise in the rate of lipolysis, although lipids are not considered to be good storage material for snails (van der Horst et al. 1974, Duncan et al. 1987). Other metabolic factors should be taken into account in the biochemical adaptation to estivation, which is a complex phenomenon involving several different physiological situations such as fasting, drought and reduced oxygen consumption. It should also be mentioned that this physiological condition must present regulatory mechanisms against oxidative stress. Studies along this line have been conducted on land snails under estivation and have revealed a higher superoxide dismutase (SOD) and glutathione peroxidase

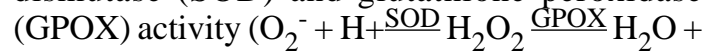
$\mathrm{O}_{2}$ ) in $O$. lactea. The increased activity of antioxidant enzymes in tissue is believed to be a mechanism of protection against oxidative stress (HermesLima \& Storey 1995a,b). A $15-30 \%$ fall in oxygen consumption does not provoke intense formation of $\left(\mathrm{O}_{2}^{-}, \mathrm{H}_{2} \mathrm{O}_{2}, . \mathrm{OH}\right)$, as reported for $O$. lactea. It is necessary to investigate the mechanism of formation of organic acids as final products, more specifically acetate, whose formation may contribute to the equilibrium of these byproducts of metabolism and of the redox system.

The low concentration of ketone bodies ( $B$ hydroxybutyrate and acetoacetate) in estivating snails indicates either an increase in ketolysis or a decrease in ketogenesis. For this reason, it is not possible to conclude whether ketone bodies provide an alternative source of energy substrates, as occurs in mammals.

\section{REFERENCES}

Barbosa FS, Barbosa I 1958. Dormancy during the larval stages of the trematode Schistosoma mansoni in snails estivating on the soil of dry natural habitats. Ecology 39: 763.

Becker W 1980. Microcalorimetric studies in Biomphalaria glabrata: The influence of Schistosoma mansoni on the Basal Metabolism. J Comp Physiol 135: 101-105.

Becker W, Lamprecht I 1977. Mikrokalorimetrische Untersuchungen zum Wirt-Parasit-Verhältnis zwischen Biomphalaria glabrata und Schistosoma mansoni. Z Parasitenkd 53: 297-305.

Bezerra JCB, Becker W, Zelck UE 1997. A comparative study of the organic acid content of the hemolymph of Schistosoma mansoni-resistant and susceptible strains of Biomphalaria glabrata. Mem Inst Oswaldo Cruz, 92: 421-425.

Brooks SPJ, Storey KB 1992. Properties of pyruvate dehydrogenase from the land snail, Otala lactea: control of enzyme activity during estivation. Physiological Zoology 65: 620-633.

Brooks SPJ, Storey KB 1996. Protein kinase involvement in land snail aestivation and anoxia: Protein kinase A kinetic properties and changes in second messenger compounds during depressed metabolism. Mol Cell Biochem 156: 153-161.

Bryant C 1993. Organic acid excretion by helminths. Parasitol Today 9: 58-60.

De Jorge FB, Petersen JA 1970. Urea and uric acid contents in the hepatopancreas, kidney and lung of active and dormant snails, Strophochelius and Thaumastus (Pulmonata; Mollusca). Comp Biochem Physiol 32: 211-219.

Duncan M, Fried B, Sherma J 1987. Lipids in fed and starved Biomphalaria glabrata (Gastropoda). Comp Biochem Physiol 86A: 663-665.

Ellersiek B 1976. Auswirkung von Trockenheit auf Wirbellose. Staatsexamenarbeit Zoologisches Institut und Museum der Universität Hamburg, Germany.

Hermes-Lima M, Storey KB 1995a. Anti-oxidant defenses and metabolic depression in pulmonate land snails. Am J Physiol 261: H632-638.

Hermes-Lima M, Storey KB 1995b. Xanthine oxidase and xanthine dehydrogenase from an estivating snail. A possible role in the induction of oxidative stress. Zeitschrift für Naturforschung. C: Biosciences 50: 685-694.

Herreid CF 1977. Metabolism of land snails (Otala lactea) during dormancy, arousal, and activity. Comp Biochem Physiol 268: R1386-1393.

Hochachka PV 1983. The Mollusca. In KM Wilbur, Vol. 1, Academic Press, New York 510 pp.

Horne FR 1979. Comparative aspects of estivating metabolism in the gastropod, Marisa. Comp Biochem Physiol 64A: 309-311.

Mehlman B, von Brand T 1951. Futher studies on the anaerobic metabolism of some fresh water snails. Biol Bull 100: 199-205.

Richards CS 1967. Estivation of Biomphalaria glabrata (Basommatophora, Planorbidae). Am J Trop Med Hyg 16: 797-802.

Rumsby G, Belloque J, Ersser RS, Seakins JWT 1987. Effect of temperature and sample preparation on performance of ion-moderated partion chromatography of organic acids in biological fluids. Clin Chim Acta 163: 171-183.

Sachs L 1990. Statistische Auswertungsmethoden, Springer-Verlag, Berlim, 545 pp.

Schmidt-Nielsen K 1990. Animal Physiology, Cambridge University Press, Cambridge, 240 pp.

Standen OD 1951. Some observations upon the maintenance of Australorbis glabratus in the laboratory. Ann Trop Med Parasitol 45: 80-83.

Storey KN 1996. Oxidative stress: animal adaptations in nature. Braz J Med Biol Res 29: 1715-1733.

van der Horst DJ, Oudejans RCHM, Meijers JA, Testerink GJ 1974. Fatty acid metabolism in hibernating Cepae nemoralis (Mollusca:Pulmonata). $J$ Comp Physiol 91: 247-256.

von Brand T, Mehlman B 1953. Relation between preand post-anaerobic oxygen consumption and oxigen tension in some fresh water snails. Biol Bull 104: 301-312.

Whitwam RE, Storey KB 1990. Pyruvate kinase from the land snail Otala lactea: regulation by reversible 
784 Organics Acids in B. glabrata under Estivation - José Clecildo Barreto Bezerra et al.

phosphorylation during estivation and anoxia. $J$ Exp Biol 154: 321-337.

Wieser W 1980. Metabolic end products in three species of marine gastropods. J Mar Biol Assoc UK 60: 175-
180.

Wolmarans CT 1987. Biomphalaria glabrata: respiration, calcium and end products of carbohydrate metabolism. Comp Biochem Physiol 87A: 785-790. 